optogenetics approaches are approved for specific clinical applications, this type of closed-loop system might be poised to have a key role in driving a transformative shift towards the use of such strategies to treat human disease.

Ellen T. Roche is at the Institute for Medical Engineering and Science, Massachusetts
Institute of Technology, Massachusetts 02139, USA.

e-mail:etr@mit.edu

1. Birmingham, K. et al. Nature Rev. Drug Discov. 13, 399-400 (2014).

2. Siegel, S. W. et al. Urology 56 (6, Suppl. 1), 87-91 (2000).

3. Kavvadias, T., Huebner, M., Brucker, S. Y. \& Reisenauer, C. Arch. Gynecol. Obstet. 295, 951-957 (2017).
4. Mickle, A. D. et al. Nature 565, 361-365 (2019).

5. Krishnan, S. R. et al. Small 14, 1803192 (2018).

6. Krishnan, S. R. et al. Sci. Transl. Med. 10, eaat8437 (2018).

7. Chen, X. et al. Nature Commun. 9, 1690 (2018)

8. Nan, K. et al. Sci. Adv. 4, eaau5849 (2018).

9. Xu, L. et al. Nature Commun. 5, 3329 (2014)

10.Gustaffson, F. \& Rogers, J. G. Eur. J. Heart Failure 19 595-602 (2017).

11. Roche, E. T. et al. Sci. Transl. Med. 9, eaaf3925 (2017)

This article was published online on 2 January 2019.

\title{
Heart of a stellar explosion revealed
}

\section{During the deaths of some massive stars, a narrow beam called a jet is launched through the stellar envelope, leaving an imprint that is difficult to detect. Such an imprint has now been seen in unprecedented detail. SEE LETTER P.324}

\section{EHUD NAKAR}

$\mathrm{T}$ The death of a massive star is a spectacular event. When the star has consumed all of its nuclear fuel, its core collapses. This leads to a strong blast wave that ejects the stellar envelope at a velocity of about $1-3 \%$ of the speed of light, producing a supernova explosion ${ }^{1}$. In rare cases, the collapsing core also launches an energetic beam of matter and radiation, known as a jet, through the stellar envelope at almost the speed of light ${ }^{2}$. The jet emerges from the stellar surface and generates a bright burst of $\gamma$-rays, which can be seen only when the jet points directly towards Earth. On page 324, Izzo et al. ${ }^{3}$ report spectroscopic observations of a supernova associated with a $\gamma$-ray burst, improving our understanding of the interplay between the jet and the exploding stellar envelope.

Theory predicts that, as a jet pushes its way through a stellar envelope, it heats the gas that surrounds its path, producing a highly pressurized, hot bubble known as a cocoon ${ }^{4-6}$ (Fig. 1a). As the jet breaks out of the star, so does the $\operatorname{cocoon}^{7}$ (Fig. 1b; see also the movies at go.nature.com/2sdoeao). The cocoon carries information about the interaction between the jet and the envelope and opens a window on the inner workings of the explosion.

The predicted signature of the cocoon initially includes a relatively faint flare of $\gamma$-rays that is emitted when the cocoon breaks out of the stellar envelope ${ }^{8-10}$. This is followed by a fading signal that is seen first in X-rays and later in ultraviolet and optical light ${ }^{11}$. In addition, the cocoon spreads sideways, engulfing the expanding envelope so that the supernova light must pass through the cocoon material on its way to Earth. During the first few days after the explosion, this passage leaves an imprint of broad absorption features on the spectrum of light from the supernova ${ }^{12}$. Later, as the cocoon expands, it becomes fully transparent, and the absorption features disappear.

For decades, astronomers have been observing the emissions from ejected envelopes and from jets - the supernovae and the $\gamma$-ray bursts, respectively. However, although cocoons are predicted to transport as much energy as the other two components ${ }^{11}$, identifying their observational signatures turned out to be extremely difficult. When a jet is pointing towards Earth, the $\gamma$-ray burst and the afterglow of the burst outshine the cocoon emission, as well as the supernova light, for several days. And when a jet is pointing away from Earth, the supernova is usually detected too late, after the absorption signal has disappeared. Izzo and colleagues present observations of an event in which a $\gamma$-ray burst is seen, but the afterglow of the burst is faint enough to

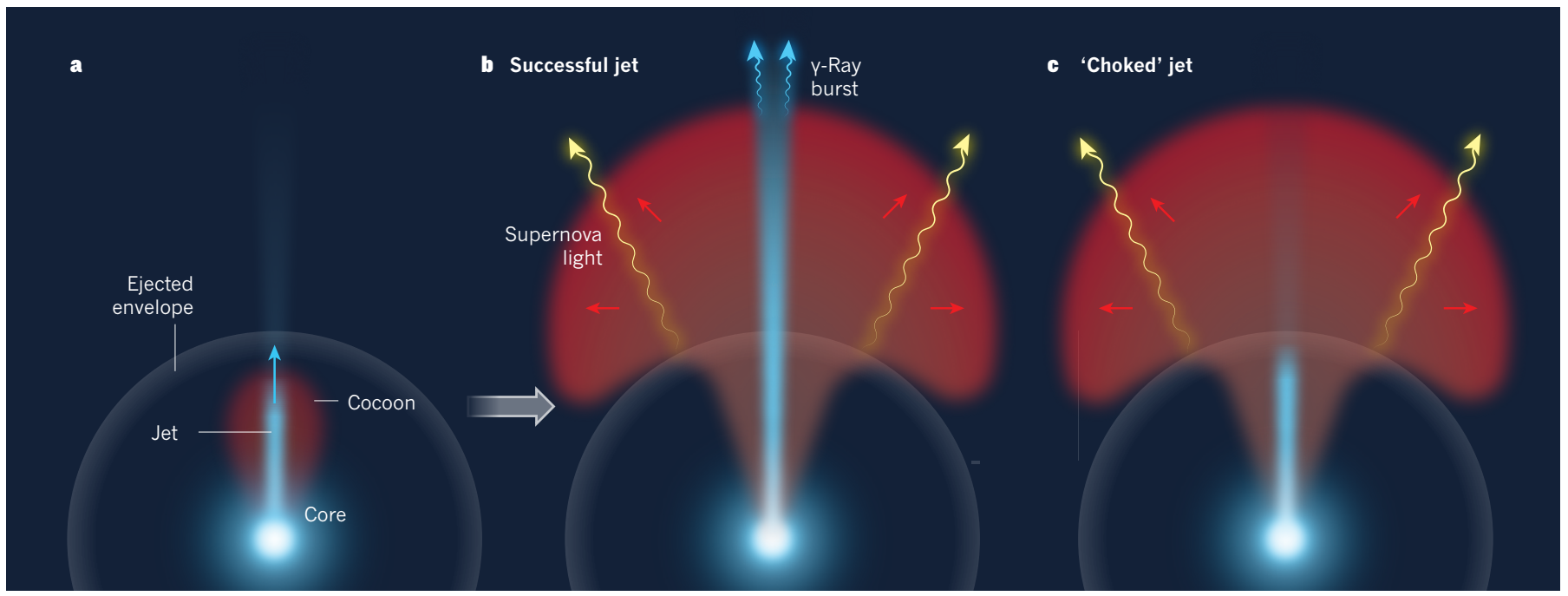

Figure 1 | Jets and cocoons in supernova explosions. a, During the death of a massive star, the stellar envelope is ejected to produce a supernova. In rare cases, the core of the star launches (blue arrow) a narrow beam of matter and radiation called a jet through the envelope, generating a hot bubble known as a cocoon. Izzo et al. ${ }^{3}$ report observations of such a cocoon in a particular supernova. $\mathbf{b}$, If the jet successfully breaks out of the stellar envelope, it produces a bright burst of $\gamma$-rays. The cocoon also breaks out, and spreads sideways (red arrows) to engulf the star. Supernova light from the envelope is partially absorbed as it passes through the cocoon on its way to Earth. c, In other supernovae, jets can be 'choked' - they lose all of their energy while still inside the envelope. However, the cocoon can still break out and form an outflow similar to that of the cocoon of a successful jet. 
allow the cocoon to be detected within a day of the stellar explosion.

According to theoretical models ${ }^{11}$, the cocoon moves faster than the envelope, but more slowly than the jet. Moreover, the jet is expected to drag material from the core of the exploding star and to deposit it in the cocoon ${ }^{13,14}$. Indeed, Izzo et al. find broad absorption features in the supernova spectra that correspond to an outflow that is moving up to one-third the speed of light. These features indicate that the fast-moving material is substantially enriched in 'iron-peak' elements (such as iron, cobalt and nickel), which must have been produced in the stellar core during the explosion. The authors' observations provide direct evidence that material from the stellar core is dragged along by the jet and deposited in the outermost layers of the envelope.

Although the $\gamma$-ray burst itself was faint, suggesting a weak jet, the energy deposited in the cocoon revealed that the jet was highly energetic. This finding might imply that the observed faint $\gamma$-rays originate from a jet that is pointing slightly away from Earth. Alternatively, a more intriguing possibility, and one that I think is more probable, is that we are seeing the cocoon directly, and that the faint $\gamma$-rays are the predicted signal of the cocoon breaking out of the stellar envelope ${ }^{8-10}$. Evidently, Izzo and colleagues' exquisite data set carries a lot of information, and considerable theoretical work will be needed to uncover its full ramifications.

Even after decades of extensive astrophysical study, the processes by which massive stars explode are not fully understood, and the role of jets, if they have one, is unknown. Moreover, although jets are rarely observed in these explosions, it is possible that they are much more common than the observations would suggest. Jets require specific conditions to successfully cross the entire stellar envelope and break out. Most probably, many jets are 'choked' - they dissipate all of their energy while still buried in the envelope ${ }^{15}$. Although such choked jets cannot be seen directly, their cocoons can still break out (Fig. 1c; see also the movies at go.nature.com/2sdoeao), and therefore hold the key to identifying these hidden jets.

Remarkably, choked jets in supernovae might have been identified already. Broad absorption features from fast-moving material were seen in early spectra of several supernovae, and have been interpreted as the signature of jet-driven cocoons ${ }^{12}$. Most intriguingly, these features have been found not only in supernovae that are associated with $\gamma$-ray bursts, but also in several that are not ${ }^{12}$. Izzo and colleagues' findings support the interpretation of these observations as the marks of hidden jets.

The future looks promising. Next-generation wide-field optical surveys will detect many supernovae within less than a day of their explosion, enabling an effective search for the telltale cocoons. Izzo and colleagues' results pave the way for these searches, which could resolve the long-standing enigma concerning the role of jets in the explosive deaths of massive stars.

Ehud Nakar is in the Department of Astrophysics, Sackler School of Physics and Astronomy, Tel Aviv University, Tel Aviv 69978, Israel.

e-mail:udini@wise.tau.ac.il

1. Alsabti, A. W. \& Murdin, P. Handbook of Supernovae (Springer, 2017).

2. Piran, T. Rev. Mod. Phys. 76, 1143-1210 (2005).

3. Izzo, L. et al. Nature 565, 324-327 (2019).

4. Blandford, R. D. \& Rees, M. J. Mon. Not. R. Astron. Soc. 169, 395-415 (1974).

5. Mészáros, P. \& Rees, M. J. Astrophys. J. 556, L37-L40 (2001).

6. Bromberg, O., Nakar, E., Piran, T. \& Sari, R. Astrophys. J. 740, 100 (2011).

7. Ramirez-Ruiz, E., Celotti, A. \& Rees, M. J. Mon. Not. R. Astron. Soc. 337, 1349-1356 (2002)

8. Kulkarni, S. R. et al. Nature 395, 663-669 (1998).

9. Campana, S. et al. Nature 442, 1008-1010 (2006).

10.Nakar, E. Astrophys. J. 807, 172 (2015).

11.Nakar, E. \& Piran, T. Astrophys. J. 834, 28 (2017).

12.Piran, T., Nakar, E., Mazzali, P. \& Pian, E. Preprint at https://arxiv.org/abs/1704.08298 (2017).

13. Maeda, K. et al. Astrophys. J. 565, 405-412 (2002).

14. Maeda, K. \& Nomoto, K. Astrophys. J. 598, 1163-1200 (2003).

15. Bromberg, O., Nakar, E., Piran, T. \& Sari, R. Astrophys. J. 749, 110 (2012).

\section{Mutations differ in normal and cancer cells}

What determines whether genetic mutations lead to cancer? Analyses of healthy cells in the human oesophagus reveal that a high level of genetic alterations arises as people age, yet this doesn't usually result in cancer. SEE ARTICLE P.312

\section{FRANCESCA D. CICCARELLI}

$\mathrm{E}$ rrors in DNA replication can alter a cell's DNA sequence. If such alterations occur early enough in embryonic development, the changes are inherited by all of an organism's cells. But if the alterations arise later in adult life, it is more difficult to track such changes in a small number of cells in a specific tissue, so the extent of these alterations in normal tissues is poorly understood. It is thought that cancer is initiated when cells acquire a minimum compendium of genetic alterations needed to trigger tumour formation. Understanding when such initiating mutations occur in normal cells is crucial for enabling reconstruction of the early events that lead to cancer. Yokoyama et al. ${ }^{1}$, on page 312 , and
Martincorena et al. ${ }^{2}$, writing in Science, have analysed the extent of mutations in human epithelial tissue from the healthy oesophagus, and how this relates to the processes that drive cancer development.

Martincorena and colleagues sequenced 74 cancer-associated genes in 844 tissue samples taken from the upper oesophagus of 9 healthy donors who differed in gender, age and lifestyle. For 21 of these samples, the authors also determined whole-genome sequences. A previous study ${ }^{3}$ assessing mutations in healthy skin cells reported between two and six mutations per million nucleotides of DNA. By contrast, Martincorena and colleagues report that the mutations in oesophageal cells arose at a roughly tenfold lower rate than that reported for skin. This difference is unsurprising, because skin cells are exposed to more DNA-damaging agents, such as ultraviolet light, than are oesophageal cells.

Instead, the surprise is that, compared with healthy skin, the healthy oesophagus has more mutations in cancer-associated genes. Moreover, at least a subset of these altered genes was under strong positive selection, meaning that the genetic alterations promoted cell proliferation, leading to the formation of cell clones (Fig. 1). Compared with the samples from younger people, the overall number of mutations, the number of mutations in cancer-associated genes and the size of the clones were all greater in the samples from older people. The authors found that the donors' samples had an average of about 120 different mutations in NOTCH1, a known cancer-associated gene, per square centimetre of normal oesophageal tissue. Several of these mutations were of the same type as those seen in a cancer of the upper oesophagus called oesophageal squamous cell carcinoma (OSCC).

Yet despite these similarities, there were striking differences between the expansion of cell clones in the normal oesophageal tissue and in OSCC. Normal and cancerous clones seem to be driven by mutations in different genes. NOTCH1 was the most frequently mutated gene in healthy oesophageal samples, whereas a previous study ${ }^{4}$ reported 